\title{
FDLDA: An Fast Direct LDA algorithm For Face Recognition
}

\author{
GUO Zhibo, LIN Kejun \\ School of Information Engineer \\ Yangzhou University \\ Yangzhou , China \\ zhibo_guo@163.com
}

\author{
YAN Yunyang \\ Faculty of Computer Engineering \\ Huaiyin Institute of Technology \\ Huaian, China \\ areyyyke@163.com
}

\begin{abstract}
Feature extraction is one of the hot topics in face recognition. However, many face extraction methods will suffer from the "small sample size" problem, such as Linear Discriminant Analysis (LDA). Direct Linear Discriminant Analysis (DLDA) is an effective method to address this problem. But conventional DLDA algorithm is often computationally expensive and not scalable. In this paper, DLDA is analyzed from a new viewpoint via SVD and an fast and robust method named FDLDA algorithm is proposed. The proposed algorithm achieves high efficiency by introducing the SVD on a small-size matrix, while keeping competitive classification accuracy. Experimental results on ORL face database demonstrate the effectiveness of the proposed method.
\end{abstract}

Keywords-feature extraction; fast direct linear discriminant analysis;face recogniation

\section{INTRODUCTION}

Face Recognition (FR) has a wide range of applications, such as military, commercial and law enforcement et al. Within the past two decades, numerous FR [1-3],[6-20] algorithms have been proposed. Among these FR methods, the most popular methods are appearance-based approaches.

Of the appearance-based FR methods, those utilizing Linear Discriminant Analysis (LDA) [4-20] techniques have shown promising results. Conventional LDA [4,5] algorithm aims to find an optimal transformation by minimizing the within-class scatter matrix and maximizing the between class scatter matrix simultaneously. The optimal transformation is readily computed by applying the Eigen-decomposition to the scatter matrices. But an intrinsic limitation of conventional LDA is that its objective function requires the within-class scatter matrix nonsingular. For many real applications, the withinclass scatter matrix is often singular since the dimension of sample exceeds the number of sample and conventional LDA based methods suffer from the so-called "small sample size" [6-8] problem.

In the last decades, numerous methods have been proposed to solve this problem. Tian et al [9] used the pseudo inverse method by replacing inverse of within-class scatter matrix with

This work was sponsored by the Prospective Joint Research Project of Jiangsu Province (BY201506-01), Domestic Research Collaboration Innovation Project of Yangzhou (2014-8). its pseudo inverse. The perturbation method is used in [10], where a small perturbation matrix is added to within-class scatter matrix in order to make it nonsingular. Cheng et al [11] proposed the Rank Decomposition method based on successive eigen-decomposition of the total scatter matrix and the between-class scatter matrix. However, the above methods are typically computationally expensive since the scatter matrices are very large. Swets and Weng [12] proposed a two stages PCA+LDA method, also known as the Fisherface [6] method, in which PCA is first used for dimension reduction so as to make the within-class scatter matrix nonsingular before the application of LDA. By far, the PCA+LDA method is popular used. However, algorithms based on this solution may discard effective features in PCA step. To prevent this from happening, many extended LDA algorithms with null space conception were proposed. Chen et al [13] and Yang et al [14] developed DLDA algorithm for face recognition, which can effectively solve "small sample size" problem and extract optimal classification features from original samples. But conventional DLDA [13-15] algorithm is often computationally expensive and not scalable.

In this paper, first of all, we briefly recall the DLDA algorithm. Then we perform an in-depth analysis on DLDA algorithm and proposed a Fast DLDA algorithm. The utilization of the singular value decomposition (SVD)[19-20] on the small-size matrix is one of key steps. The proposed method is efficient and robust. Moreover, the theoretical foundation of the proposed method is revealed..

\section{OUTLINE OF DiRECT LDA}

Throughout the paper, $C$ denotes the number of classes, $m$ is the dimension, $N$ is the number of samples in each class, $\mu_{i}$ is the centroid of the $i$ th class, and $\mu$ is the holistic centroid of the whole data set. $S_{b}, S_{w}$ and $S_{t}$ represent between-class scatter matrix, within-class scatter matrix and total class scatter matrix, respectively.

DLDA [13-15] algorithm was proposed by Chen and Yang, which attempts to avoid the shortcomings existing in 
conventional solution to the "small sample size" problem. The basic idea behind the DLDA algorithm is that the null space of $S_{w}$ may contain effective discriminant information if the projection of $S_{b}$ is not zero in that direction, and that no effective information will be lost if the null space of $S_{b}$ is discarded. For example, assuming that $N_{b}$ and $N_{w}$ represent the null space of $S_{b}$ and $S_{w}$, respectively, the complement spaces of $N_{b}$ and $N_{w}$ can be written as $N_{b}^{\prime}=R^{n}-N_{b}$ and $N_{w}^{\prime}=R^{n}-N_{w}$. Therefore, the optimal discriminant subspace extracted by the DLDA algorithm is the intersection space $N_{b}^{\prime} \cap N_{w}$.

The difference between Chen's method and Yang's method is that Yang's method first diagonalizes $S_{b}$ to find $N_{b}^{\prime}$, while Chen's method first diagonalizes $S_{w}$ to find $N_{w}$. Although there is no significant difference between the two approaches, it may be intractable to calculate $N_{w}$ when the size of $S_{w}$ is large, which is the case in most FR application. Therefore, we adopted Chen's method as the derivation of the proposed algorithm.

\section{FDLDA ALGORITHM FOR DIMENSION REDUCTION AND FEATURE EXTRACTION}

In this section, we will present the FDLDA algorithm. Based on the analysis of Chen's DLDA algorithm.

We analysis the Chen's algorithm about DLDA. They perform the Eigen value decomposition of $S_{w}$ as $S_{w}=V \Lambda V^{T}$ and let $V=\left[v_{1}, v_{2}, \cdots v_{r}, v_{r+1}, \cdots, v_{n}\right]$, then they let $Q_{\perp}=\left[v_{1}, v_{2}, \cdots v_{r}\right]$ and $Q=\left[v_{r+1}, \cdots, v_{n}\right]$ and the null space of $S_{w}$ can be spanned by $v_{r+1}, \cdots, v_{n}$, where $r$ is rank of $S_{w}$ and $n$ is the dimensionality of the feature vectors.

They Compute $\tilde{S}_{b}$ according to $\tilde{S}_{b}=Q Q^{T} S_{b} Q Q^{T}$ and calculate the eigenvectors $u_{1}, u_{2}, \cdots, u_{d}$ corresponding to the set of the largest eigenvalues of $\tilde{S}_{b}$ and use them to form the most discriminant vector set for LDA.

Due to $r<<n$, so the calculation of the $S_{w}, \mathrm{Q}$ and $\tilde{S}_{b}$ will greatly improve the computational complexity . our thoughts are calculating the final differential vector according to $Q_{\perp} \cdot Q_{\perp}$ can be obtained by solving the eigen problem on $S_{w}$ similar to PCA algorithm based SVD.

Because between-class scatter matrix $S_{b}$ and within-class scatter matrix $S_{b}$ can represented by following:

$$
S_{b}=H_{b} H_{b}^{T}, S_{w}=H_{w} H_{w}^{T}
$$

$H_{b}, \quad H_{w}$ as following:

$$
\begin{gathered}
H_{b}=\frac{1}{\sqrt{c}}\left(\bar{x}_{1}-\bar{x}, \bar{x}_{2}-\bar{x}, \cdots, \bar{x}_{c}-\bar{x}\right) \\
H_{w}=\frac{1}{\sqrt{N}}\left(x_{1}^{(l)}-\bar{x}_{1}, \cdots, x_{l}^{\left(n_{1}\right)}-\bar{x}_{1}, \cdots, x_{c}^{(l)}-\bar{x}_{c}, \cdots, x_{c}^{\left(n_{c}\right)}-\bar{x}_{c}\right)
\end{gathered}
$$

Assuming that $G_{w}=H_{w}^{T} H_{w}$, and making the eigenvalue decomposition of $G_{w}$. We can calculate the eigenvectors $z_{1}, z_{2}, \cdots, z_{d}$ corresponding to the nonzero eigenvalues $\lambda_{1}, \lambda_{2}, \cdots, \lambda_{r}$ of $G_{w}$. According eq. 4, we can get the feature vector set of $Q_{\perp}=\left(v_{1}, \cdots, v_{r}\right)$ corresponding to nonzero eigenvalues $\lambda_{1}, \lambda_{2}, \cdots, \lambda_{r}$ 。

$$
v_{i}=\frac{1}{\sqrt{l_{i}}} H_{w} z_{i}, \quad i=1, L, r
$$

$Q$ is the null space matrix of $S_{w}$. we can obtain:

$$
\tilde{S}_{b}=Q Q^{T} H_{b}\left(Q Q^{T} H_{b}\right)^{T}=\tilde{H}_{b} \tilde{H}_{b}^{T}
$$

We can construct matrix $\tilde{G}_{b}=\tilde{H}_{b}^{T} \tilde{H}_{b}$, and calculate the eigenvectors $z_{1}, z_{2}, \cdots, z_{d}$ corresponding to the set of the largest eigenvalues $\lambda_{1}, \lambda_{2}, \cdots, \lambda_{d}$ of $\tilde{G}_{b}$, therefore the eigenvectors $u_{1}, u_{2}, \cdots, u_{d}$ corresponding to the set of the largest eigenvalues of $\tilde{S}_{b}$ can be calculated by the following equation :

$$
u_{i}=\frac{1}{\sqrt{\lambda_{i}}} \tilde{H}_{b} z_{i}, \quad i=1, \cdots, d
$$

According to specialty of orthal vectors, we can obtain:

$$
Q Q^{T}=I-Q_{\perp} Q_{\perp}^{T}
$$

According to eq.7, we have:

$$
\tilde{H}_{b}=Q Q^{T} H_{b}=H_{b}-Q_{\perp} Q_{\perp}^{T} H_{b}
$$

Therefore, we can get:

$$
\begin{aligned}
& \tilde{G}_{b}=\left(H_{b}-Q_{\perp} Q_{\perp}{ }^{T} H_{b}\right)^{T}\left(H_{b}-Q_{\perp} Q_{\perp}{ }^{T} H_{b}\right) \\
& =H_{b}{ }^{T} H_{b}-H_{b}{ }^{T} Q_{\perp} Q_{\perp}{ }^{T} H_{b} \\
& =H_{b}{ }^{T} H_{b}-\left(Q_{\perp}{ }^{T} H_{b}\right)^{T} Q_{\perp}{ }^{T} H_{b}
\end{aligned}
$$

According to eq.9, $\tilde{G}_{b}$ can be calculated in a lowdimensional space, which greatly saves storage space and reduces the amount of computation. For example, the original 
sample dimension $M=10304$, the number of categories $c=40$ in the ORL face database. if we choose 200 training samples, the rank r of $S_{w}$ is 160 . the vector number of $Q_{\perp}$ is 160 , but the vector number of $Q_{\perp}$ is up to 10304 . The memory space of $Q$ is dozens of times of $Q_{\perp}$

Based on the above discussion, the proposed FDLDA algorithm is described as follows:

Step 1: Obtain $H_{w}, H_{b}$ and calculate $Q_{\perp}$ based on SVD. Let $\tilde{G}_{b}=H_{b}{ }^{T} H_{b}-\left(Q_{\perp}{ }^{T} H_{b}\right)^{T} Q_{\perp}{ }^{T} H_{b}$.

Step 2: Calculate the eigenvector matrix $Z=\left[z_{1}, z_{2}, \cdots, z_{d}\right]$ and the eigenvectors $z_{1}, z_{2}, \cdots, z_{d}$ corresponding to the set of the largest eigenvalues $\lambda_{1}, \lambda_{2}, \cdots, \lambda_{d}$ of $\tilde{G}_{b}$.

Step 3: According to $u_{i}=\frac{1}{\sqrt{\lambda_{\imath}}}\left(H_{b}-Q_{\perp} Q_{\perp}{ }^{T} H_{b}\right) z_{i}, i=1, \cdots, d$ Calculate the eigenvector matrix $U=\left[u_{1}, u_{2}, \cdots, u_{d}\right]$ and the discriminant matrix $A=U-Q_{\perp} Q_{\perp}{ }^{T} U$.

Step 4: Using the discriminant matrix $A$ extract efficient features and classify.

\section{EXPERIMENTAL RESULTS}

To demonstrate the effectiveness of our method, experiments were done on the ORL face database. Fig.1 depicts some images from the ORL face database. ORL database includes 200 samples (40 individuals, each 10 images).

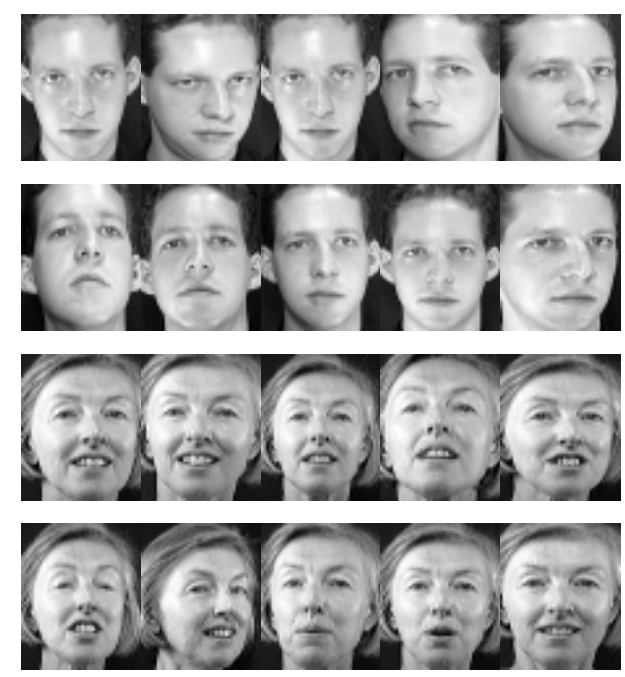

Fig. 1. Some samples from the ORL face database

For comparison of FDLDA anf Chen's DLDA algorithm, the experiment uses three resolutions $(46 \times 56,35 \times 42,23 \times 28)$. the sample image is obtained by bilinear interpolation. In our experiments, the training and testing set are selected randomly from each subject. In each round, the training samples are selected randomly from the gallery and the remaining samples are used for testing. This procedure was repeated 10 times by randomly choosing different training and testing sets. After feature extraction, a nearest neighbor classifier with different distance metrics is employed for classification.

Experimental environment: MATLAB2010, Core i5, SRAM 2G and so on.

Tabel 1. The experimental Comparison using $45 \times 56$ resolution

\begin{tabular}{lcccc}
\hline & FDLDA & & Chen's DLDA \\
\cline { 2 - 2 } Recognition rate(\%) & 95.0 & & 95.0 \\
Trainning time (s) & 2.3 & & 552 \\
\hline
\end{tabular}

Tabel 2. The experimental Comparison using $35 \times 42$ resolution

\begin{tabular}{lccc}
\hline & FDLDA & & Chen’s DLDA \\
\cline { 2 - 2 } Recognition rate(\%) & 95.25 & & 95.25 \\
Trainning time (s) & 1.3 & & 88 \\
\hline
\end{tabular}

Tabel 3. The experimental Comparison using $23 \times 28$ resolution

\begin{tabular}{lcccc}
\hline & FDLDA & & Chen's DLDA \\
\cline { 2 - 2 } Recognition rate(\%) & 95.5 & & 95.5 \\
Trainning time (s) & 0.6 & & 5.4 \\
\hline
\end{tabular}

The experimental results of FDLDA algorithm and Chen's DLDA algorithm is shown on the Tab.1 Tab3. The accuracy of two algorithms is same using the same kind of resolution, but the FDLDA algorithm is much faster than Chen's DLDA on the training time. Especially with the resolution increases, the gap between training time is also growing. For different resolutions, FLDA's training time is similar and the difference is not obvious. Experimental results show that the proposed algorithm effectively solves an important problem about DLDA algorithm.

\section{CONCLUSIONS}

In this paper, we proposed an fast direct linear discriminant analysis algorithm, namely, FDLDA algorithm, which is highly efficient. The proposed method does not require the whole data matrix in main memory. This is desirable for large data sets. In addition, our theoretical analysis indicates that the computational complexity of the FDLDA algorithm is linear in the number of the data items in the training data set as the number of classes and the number of dimensions. It is the 
calculated $Q_{\perp}$ based on SVD that contributes to the efficiency of the FDLDA algorithm, which is not only shown by our theoretical analysis, but also strongly supported by our experimental results.

Our experiments on face database have shown that the accuracy achieved by the FDLDA algorithm is competitive with the ones achieved by Chen's DLDA. With efficiency and scalability, FDLDA algorithm is promising in real-time application involving extremely high-dimensional data.

\section{REFERENCES}

[1] Samal A and Iyengar P A. (1992) Automatic recognition and analysis of human faces and facial expressions: a survey. Pattern Recognition 25(1): $65-77$

[2] W. Zhao, R. Chellappa, and J. Phillips (1999) Subspace linear discriminant analysis for face recognition. Technical Report, CSTR4009, Univ. of Maryland

[3] W.Zhao, R.Chellappa, P.J.Phillips, A.Rosenfeld. (2003) Face recognition: A literature survey. ACM Computing Surveys. 35(4): 395458

[4] K.Fukunaga (1990) Introduction to statistical pattern recognition. Academic Press, Boston, 2nd edition

[5] 5. R. Fisher (1936) The use of multiple measures in taxonomic problems. Ann. Eugenics. 7:179-188

[6] 6. P. N. Belhumeur, J. P. Hespanha, and D. J. Kriengman (1997) Eigenfaces vs. Fisherfaces: Recognition using class specific linear projection. IEEE Trans. Pattern Analysis and Machine Intelligence. 19 (7): 711-720

[7] 7. J. Yang, A.F. Frangi, J.Y. Yang, D. Zhang, Z. Jin (2005) KPCA plus LDA: A Complete Kernel Fisher Discriminant Framework for Feature extraction and Recognition. IEEE Trans. Pattern Analysis and Machine Intelligence 27(2): 230-244
[8] 8. J. Yang, J.Y. Yang (2003) Why can LDA be performed in PCA transformed space?. Pattern Recognition 36:563-566

[9] 9. Q.Tian, M.Barbero, Z.Gu, S.Lee (1986) Image classification by the foley-sammon transform. Opt. Eng. 25(7):834-840

[10] 10. Z.Q.Hong, J.Y.Yang (1991) Optimal discriminant plane for a small number of samples and design method of classifier on the plane. Pattern Recognition 24(4):317-324

[11] Y.Q. Cheng, Y.M.Zhuang, J.Y.Yang (1992) Optimal fisher discriminant analysis using the rank decomposition. Pattern Recognition 25(1):101111.

[12] Swets and J. Weng (1996) Using discriminant eigenfeatures for image retrieval. IEEE Trans. on Pattern Analysis and Machine Intelligence 18(8):831-836.

[13] L.F.Chen, H.Y.M.Liao, J.C.Lin, M.T.Ko, and G.J.Yu. A New LDAbased Face Recognition System Which Can Solve the Small Sample Size Problem, Pattern Recognition, 2000, 33(10):1713-1726.

[14] J. Yang, H. Yu, W. Kunz (2000) An Efficient LDA Algorithm for Face Recognition. International Conference on Automation, Robotics, and Computer Vision (ICARCV'2000), Singapore, December.

[15] Yujie Zheng, Jingyu Yang, Jian Yang, Xiaojun Wu (2006) Effective classification image space which can solve small sample size problem. Proc. Of. the 18th Int. Conf. on Pattern Recognition (ICPR'06), vol.3, pp.861-864.

[16] Z. Wang, Q. Ruan, G. An (2016) Facial expression recognition using sparse local Fisher discriminant analysis, Neurocomputing, 2016(174): 756-766.

[17] M. Kan, S. Shan, H. Zhang, S. Lao (2016) X ChenMulti-view Discriminant Analysis, IEEE Trans. on Pattern Analysis and Machine Intelligence, 38(1):808-821.

[18] J. Yang, Z. Gu, N. Zhang, J. Xu (2014) Median-mean line based discriminant analysis, Neurocomputing, 123: 233-246.

[19] Z. Q. Hong (1991) Algebraic feature extraction of image for recognition, Pattern Recognition, 24(3): 211-219.

[20] W. Y Pang, N. H Yu, R. Zhang (2004) Fusion of SVD and LDA for face recognition, In Proceedings of the lntemational Conference on Image Processing, Singapore,2: 1417-1420. 